\title{
Explicit multi-model predictive control of a waste heat Rankine based system for heavy duty trucks
}

\author{
Vincent Grelet ${ }^{1,2,3}$, Pascal Dufour ${ }^{2, \star}$, Madiha Nadri ${ }^{2}$, Vincent Lemort ${ }^{3}$ and Thomas Reiche ${ }^{1}$
}

\begin{abstract}
This paper presents an explicit multi-model predictive controller (MMPC) for a waste heat recovery system (WHRS) mounted on a heavy duty truck engine. WHRS based on the Rankine cycle principle attracts a lot of interest in the heavy duty industry, over the past few years, to decrease the fuel consumption and reach the future pollutant emissions standards. Control issues have still to be faced before the integration of such a system into a vehicle. Model predictive controllers suits really well for our control purpose due to their ability to handle online optimal control problem. This paper focuses on the development of an explicit control law based on MMPC with aim to control the fluid temperature at the inlet of the expansion machine in order to increase the system performance and the reliability.
\end{abstract}

\section{INTRODUCTION}

Over the last few years, the increase in fuel price coupled to the more and more stringent pollutant emissions legislations have compelled the truck manufacturers to look into innovative solutions to decrease the fuel consumption. Waste heat recovery systems (WHRS) such as Rankine cycle could be one of those solutions [1]. Recovering waste heat by the way of a Rankine cycle and turned it into another form of energy is not really new. This principle is used for electricity generation for years since today a large proportion of the worldwide electricity production is based on the Rankine process using a wide variety of heat sources. In the last decades, automotive industry has been placing more and more attention in recovering waste heat. The most applied system, today, is the turbocharger which turns available energy into aeraulic work. Another example is the turbo-compound which transforms wasted energy into mechanical one. In addition to those systems, energy recovery based on the Rankine cycle has attracted a lot of interest over the last years and a significant potential has been shown in heavy duty (HD) industry and especially for long haul truck applications [2], [3]. If working fluid (WF), heat source, heat sink and components choice are largely addressed topics, only few papers treat of control system development [4], [5]. To ensure safe operation and improve the operating strategy, a key variable is the working fluid

\footnotetext{
${ }^{1}$ Volvo Trucks Global Trucks Technology Advanced Technology and Research, 1 av Henri Germain 69800 Saint Priest, France vincent.grelet@volvo.com, thomas.reiche@volvo.com

${ }^{2}$ Université de Lyon, F-69622, Lyon, France - Université Lyon 1, Villeurbanne, France - CNRS, UMR 5007, LAGEP, France. dufouralagep.univ-lyon1.fr, nadrielagep. univ-lyon 1.fr

${ }^{3}$ LABOTHAP, University of Liege, Campus du Sart Tilman Bat. B49 B4000 Liege, Belgium. vincent. lemort@ulg.ac.be

$\star$ Corresponding author.
}

temperature at the expansion machine inlet: This temperature is often turned into a superheat criterion (superheat is the temperature difference between the actual value and the saturation temperature) and needs to be accurately controlled in order to maximize the power production and increase the power generation period by feeding the expander with vapor at good conditions. Moreover, technical integration constrains that the control algorithm has to be yet simple to limit the computational complexity (attribute a large part of the computational capability of the electronic control unit to a classical optimization problem is not possible) and fast to compute (sampling time is 20ms). This paper is organized as follows: section 2 presents the principle and the studied system are explained. Section 3 approaches the controller methodology development when section 4 shows some numerical results. Finally a conclusion is drawn.

\section{PRINCIPLE OF OPERATION AND STUDIED SYSTEM}

\section{A. Rankine process}

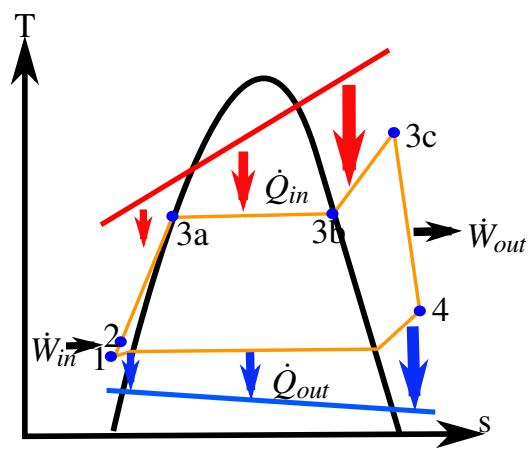

Fig. 1. Temperature-entropy diagram of the Rankine cycle

Rankine cycle process is well known and widely used for years. It relies on four transformations and is a variant of the Carnot cycle. First, the fluid is pumped and compressed from the condensing pressure to the evaporation pressure $(1 \rightarrow 2)$. The pressurized liquid goes through one or several heat exchangers to recover thermal power $\left(\dot{Q}_{i n}\right)$ from the heat source(s) to be preheated $(2 \rightarrow 3 a)$, vaporized $(3 a \rightarrow 3 b)$ and then superheated $(3 b \rightarrow 3 c)$. This superheated vapor is expanded $(3 c \rightarrow 4)$ through the expander which aims to convert the pressure drop of the expansion from evaporating to condensing pressure into a mechanical power on its shaft $\left(\dot{W}_{\text {out }}\right)$. Finally the fluid at low pressure, which can be fully vapor or partially condensed after the expansion process (depending on the technology used and the superheat level 
at expander inlet), is condensed (i.e. changes from vapor to liquid phase $4 \rightarrow 1$ ) releasing heat $\left(\dot{Q}_{\text {out }}\right)$ into the heat sink. An usual representation of the thermodynamic cycle is done through a temperature-entropy (T-s) form: Figure 1 shows the state changes of the working fluid (or Rankine fluid) and transfer fluids (heat source and sink) of a subcritical Rankine cycle.

\section{B. Studied system}

The studied WHRS (Figure 2) recovers heat from both exhaust gas recirculation (EGR) and exhaust in series, which means that the working fluid goes first through the evaporator linked to the EGR and then through the tailpipe boiler (linked to the exhaust gases) [6]. This special configuration has been chosen to lower the EGR temperature downstream the boiler. The Rankine fluid is pumped in an atmospheric vented tank and pressurized by the feed pump. By controlling its speed, the working fluid mass flow rate going through the evaporators is set. The fluid then flows through the EGR and the tailpipe boiler where it is turned into vapor. It is expanded in a turbine, condensed in a water cooled condenser and goes back to the tank. The system is mounted onto an $11 \mathrm{~L}$ 6 cylinder turbocharged engine with diesel particulate filter (DPF) and selective catalyst reduction (SCR) exhaust after treatment system. The working fluid is a mixture of water and ethanol which reduces the flammability of pure ethanol and decreases the freezing temperature of pure water.

\section{RANKINE CYCLE CONTROLLER}

\section{A. Controller aim}

This paper describes a temperature controller development for the system mentioned in section I-B. The aim is to reduce the standard variation around the set point. To do that, the manipulating variable (MV) is the working fluid mass flow rate, and is used to ensure that the temperature after the two evaporators (the controlled variable $\mathrm{CV}$ ) track a desired set-point. In the following, superheat is used instead of temperature since only normal operation mode, where the goal is to keep the working fluid in vapor phase, is considered. Having an efficient control of it is important to maximize the system efficiency and to ensure that no liquid enters in the turbine which can be destroyed due to its high rotational speed. This work focuses only on the evaporators models since the system dynamic is mainly controlled by the heat exchangers (HEX) behavior.

Moreover, technical integration impacts the choice of the control algorithm: it has to be simple (poor computational capability) and fast to be computed (sampling time is 20ms).

\section{B. Nonlinear evaporator model}

The input vector $u$ contains the MV $\dot{m}_{f_{0}}$ and four inputs disturbances which are: the WF pressure and enthalpy entering in the evaporator $\left(P_{f_{0}}\right.$ and $\left.h_{f_{0}}\right)$ and the gas mass flow and

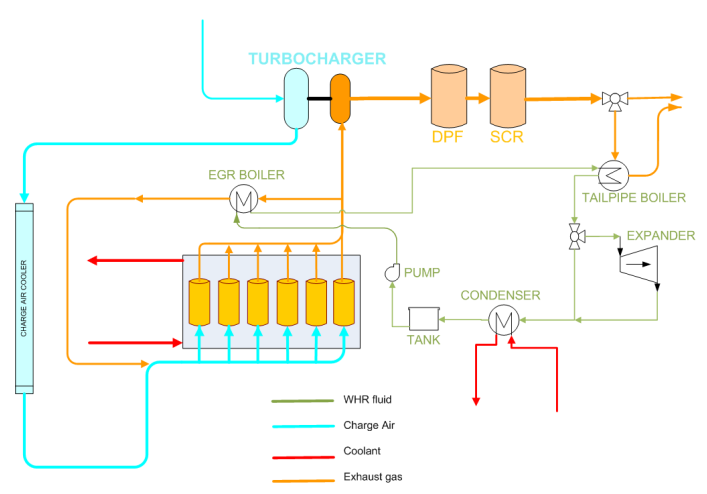

Fig. 2. Studied system schematic

temperature entering in the boiler (respectively $\dot{m}_{g_{L}}$ and $T_{g_{L}}$ ). The state vector contains: the WF mass flow and enthalpy (respectively $\dot{m}_{f}$ and $h_{f}$ ), internal wall temperature $\left(T_{w_{\text {int }}}\right)$, transfer fluid temperature $\left(T_{g}\right)$ and external wall temperature $\left(T_{w_{\text {ext }}}\right)$. The complete model development and experimental validation is shown in [6]. After the spatial discretization, the system of equations defining the response of the $i^{t h}$ cell for working fluid, internal pipe, transfer fluid and external pipe is shown by equation (1):

$$
\begin{aligned}
Z \dot{x}_{i} & =f_{i}(x, u) \\
\text { where: } Z & =\left[\begin{array}{lllll}
0 & 0 & 0 & 0 & 0 \\
0 & 1 & 0 & 0 & 0 \\
0 & 0 & 1 & 0 & 0 \\
0 & 0 & 0 & 1 & 0 \\
0 & 0 & 0 & 0 & 1
\end{array}\right] \\
x_{i}^{T} & =\left[\begin{array}{lllll}
\dot{m}_{f_{i}} & h_{f_{i}} & T_{w_{\text {int }_{i}}} & T_{g_{i}} & T_{w_{\text {ext }}}
\end{array}\right] \\
u^{T} & =\left[\begin{array}{lllll}
\dot{m}_{f_{0}} & P_{f_{0}} & h_{f_{0}} & \dot{m}_{g_{L}} & T_{g_{L}}
\end{array}\right]
\end{aligned}
$$

$f_{i}\left(x_{i}, u\right)=$

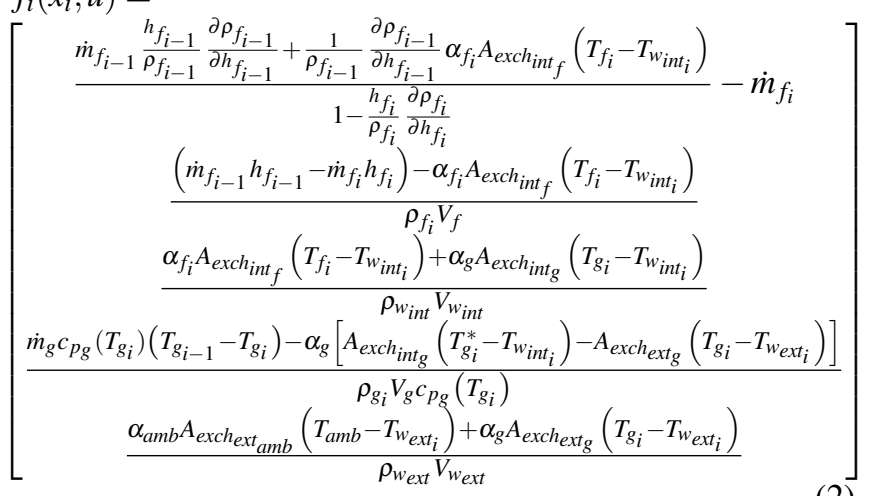

where $i \in\left[1: n_{d}\right]$ and the ambient temperature $T_{a m b}$ is taken constant around the entire HEX.

\section{Local multi linear model development}

Development of a good nonlinear first principle model is a severe effort (many parameters are usually hard to get from experimental results) and may be difficult to be implemented in an online model based control strategy. Here, integration constrains lead to develop a simple and fast controller. In that case, many works report the usage of multi linear model 
approaches [7], [8]. Let us consider the single input single output (SISO) nonlinear model of the form:

$$
\begin{aligned}
& \dot{x}=f(x, u), \\
& y=g(x, u),
\end{aligned}
$$

where the input is the considered MV (the WF mass flow rate entering in the EGR boiler) and the output is the considered $\mathrm{CV}$ (the WF temperature at the outlet of the tailpipe boiler). The four remaining input components in (1) are further considered as input disturbances. By plant experiments or simulation of the detailed non linear model (1-2), the relationship between the MV and the CV defined in the nonlinear SISO system (3-4) can be identified by a series of first order plus time delay (FOPTD) models (each with a subscript $i \in[1, N]$ ), where the model parameters are the static gain $G_{i}$, the time constant $\tau_{i}$ and the delay $L_{i}$ :

$$
F_{i}(p)=\frac{y_{i}}{u}=\frac{G_{i}}{1+\tau_{i} p} e^{-L_{i} p} .
$$

In the sequel, the scalar MV is $u$ and the scalar $\mathrm{CV}$ is $y_{i}$. To obtain a global model, theses $N$ local models have now to be combined. The weighting scheme has to estimate the probability of one model among $N$ to be valid by comparing the process measurements to the output of each local models. The global model output is then calculated by weighting the local model outputs and sum them.

\section{Weighting schemes}

The two weighting schemes, used in the latter, estimate the validity of the $i^{\text {th }}$ model by calculating the modeling error:

$$
\varepsilon_{i, k}=y_{p, k}-y_{i, k}
$$

which corresponds to the $i^{\text {th }}$ model error at the current time $t_{k}$ between the real measure $y_{p, k}$ and the $i^{\text {th }}$ model output $y_{i, k}$.

1) Bayesian estimator: Most of the study on multi linear approach found in the literature use the Bayesian estimator as adaptive estimation technique to select the best model at each time step [9], [8]. It is based on probabilities calculation $P(i)$, representing the probability of the $i^{\text {th }}$ model to be valid. This weighting scheme assigns a value between 0 and 1 to each model outputs where the weights sum is equal to 1 to ensure that the global model is always bounded by the extreme values of the $N$ models in the bank. It also allows an exact, if it appears, model to be the only prediction model. The recursive Bayesian weighting scheme is a conditional probability of the $i^{\text {th }}$ model among the $N$ models of the bank to be true given the model population in the bank and its past history of probabilities. It is written as follows:

$$
\begin{aligned}
& p_{i, k}=\frac{\exp \left(-\frac{1}{2} \varepsilon_{i, k} K_{i} \varepsilon_{i, k} p_{i, k-1}\right)}{\sum_{m=1}^{N}\left(\exp \left(-\frac{1}{2} \varepsilon_{m, k} K_{m} \varepsilon_{m, k} p_{m, k-1}\right)\right.}, \\
& w_{i, k}= \begin{cases}\frac{p_{i, k}}{\sum_{m=1}^{N} p_{m, k}} & \text { for } p_{i, k}>\delta \\
0 & \text { for } p_{i, k}<\delta .\end{cases}
\end{aligned}
$$

In the probability calculation of equations (7-8), a vector $(K)$ containing convergence factor $K_{i}(i \in[1 N])$ is used to improve the convergence to a single model. If more combination of the different models contained in the bank is desired, this factor should be detuned and set at a low value. To keep every models of the bank alive, an artificial probability is introduced such as all $p_{i, k}$ never goes below this artificial lower limit $\delta$ (which is a tuning parameter). This one is called in the following cut-off probability and is set to improve the estimator performance. However every models having this lower saturation value as probability are excluded from the weight computation as it is shown in equation (8). The model output is then defined as at the current time $t_{k}$ :

$$
y_{k}=\sum_{i=1}^{N} w_{i, k} y_{i, k} .
$$

2) New developed estimator: To simplify the weighting scheme and reduce the number of setting parameters, a weighting scheme has been developed. All values denoted by a superscript, $\sim$, refer to normalized values.

$$
\begin{aligned}
\tilde{\varepsilon}_{i, k} & =\frac{\varepsilon_{i, k}^{2}}{\sum_{m=1}^{N} \varepsilon_{m, k}^{2}}, \\
c_{i, k} & =\left(1-\tilde{\varepsilon}_{i, k}\right) \prod_{j \neq i, j=1}^{j=N} \tilde{\varepsilon}_{j, k}, \\
\tilde{c}_{i, k} & =\frac{c_{i, k}}{\sum_{m=1}^{N} c_{m, k}} .
\end{aligned}
$$

The value $\tilde{c}_{i, k}$ computed thanks to equation (12) is then filtered through a first order transfer function with unit static gain

$$
w_{i}(s)=\frac{1}{1+\tau_{\text {filt }} s} \tilde{c}_{i}(s)
$$

to obtain the current weight of each local model $w_{i, k} . \tau_{f i l t}$ is a scalar which plays a similar role as the convergence matrix $K$ used in Bayesian approach (7). The number of tuning parameters is then decreased to the only parameter $\tau_{\text {est }}$ instead of $N+1$ ( $K$ and $\lambda$ ) in the Bayesian recursive scheme. The global model output is then calculated in the same way than with the Bayesian method with (9).

\section{E. MMPC strategy}

At time $t_{k}$ (the continuous time is discretized at the constant sampling rate $T_{s}$ ), the model outputs $y_{i, k}$ of the $N$ FOPTD models (5) allow to compute the models weights $w_{i, k}$ and the final model output $y_{k}$ with (9). This modeling (5) and (9) is used in a MMPC framework to ensure a tracking of the process output $y_{p}$ to the set-point $y^{s p}$, accounting for the control move effort $\Delta u$. Since here, a major issue for the technical integration, is to decrease the computational load, the number of optimization arguments (i.e., the control horizon) is first set to its minimum: $1\left(u(t)=u\left(t_{k}\right)=u_{k}\right)$. The control horizon is $t_{p}$. Therefore, the MMPC algorithm 
aims to find, at each current time $t_{k}$, the optimal step input $u_{k}^{\star}$ that minimizes following the cost function $J$ :

$$
\left\{\begin{aligned}
\min _{u_{\text {inf }} \leq u_{k} \leq u_{\text {sup }}} J\left(u_{k}\right) & =\int_{t_{k}}^{t_{k}+t_{p}}\left(y_{p}(t)-y^{s p}\right)^{2}+w_{u} \Delta u_{k}^{2} d t, \\
\Delta u_{k} & =u_{k}-u_{k-1},
\end{aligned}\right.
$$

where $w_{u}$ is a scaling factor and a penalty weight for the control move. In this quadratic form, the ideal optimal solution leads to $J\left(u_{k}^{\star}\right)=0$, i.e.: the tracking is perfect $\left(y_{p}(t)=y^{s p} \forall t\right)$ and the control is at a steady state $\left(\Delta u_{k}=0 \forall t\right)$.

In the internal model control structure, the control $u$ is both applied to the process and the model(s). Hence, it allows to compute the modeling error $e$ at each $k$ :

$$
e_{k}=y_{p, k}-y_{k} \text {. }
$$

this error is assumed constant into the future and used to get the output prediction $y_{p}(t)$ in (14) using the model:

$$
y_{p}(t)=y(t)+e_{k} .
$$

In the same way, the set point $y^{s p}$ is taken constant on the prediction horizon and updated at each time $k$. The question is now to write $y(t)$ according to $u_{k}$ (and also the constant values and the parameters updated at each $k$ ). Let us start from the definition of the output response $y_{i}(t)$ of one linear FOPTD model $i$ (5) with any input profile $u(t)$ from any current time $t_{k}$, and starting from the current process output $y_{p, k}:$

$$
y_{i}(t)=y_{p, k} e^{\frac{-\left(t-t_{k}\right)}{\tau_{i}}}+\int_{t_{k}}^{t}\left(e^{\frac{-(t-s)}{\tau_{i}}} \frac{G_{i}}{\tau_{i}} u\left(s-\tau_{i}\right)\right) d s
$$

(17) can be developed as:

$$
y_{i}(t)=y_{p, k} e^{\frac{-\left(t-t_{k}\right)}{\tau_{i}}}+\frac{G_{i}}{\tau_{i}} e^{\frac{-t}{\tau_{i}}} \int_{t_{k}}^{t}\left(e^{\frac{s}{\tau_{i}}} u\left(s-\tau_{i}\right)\right) d s
$$

To proceed, we need to define, based on the time delay $L_{i}$ :

$$
\left\{\begin{aligned}
\lambda_{i} & =\max \left(a_{i} \in \mathbb{N} \mid a_{i} \leq \frac{L_{i}}{T_{s}}\right) \\
\Delta L_{i} & =L_{i}-\lambda_{i} T_{s}, \in \mathbb{R}^{+}
\end{aligned}\right.
$$

which allows to define $u($.$) used in the integration interval$ of (18), based on past input values and the future input value of $u$. Then, the integration in (18) is done by parts, where the $\lambda_{i}+2$ time intervals are given in table $\mathrm{I}$ :

\begin{tabular}{l|l|l}
\hline$s$ & $s-L_{i}$ & $u\left(s-L_{i}\right)$ \\
\hline$t_{k} \rightarrow t_{k}+\Delta L_{i}$ & $t_{k}-L_{i} \rightarrow t_{k-\lambda_{i}}$ & $u\left(t_{k-\lambda_{i}-1}\right)$ \\
$t_{k}+\Delta L_{i} \rightarrow t_{k}+\Delta L_{i}+T_{s}$ & $t_{k-\lambda_{i}} \rightarrow t_{k-\lambda_{i}+1}$ & $u\left(t_{k-\lambda_{i}}\right)$ \\
$\ldots$ & $\ldots$ & $\cdots$ \\
$t_{k}+\Delta L_{i}+\left(\lambda_{i}-j\right) T_{s} \rightarrow t_{k}+\Delta L_{i}+\left(\lambda_{i}-j+1\right) T_{s}$ & $t_{k-j} \rightarrow t_{k-j+1}$ & $u\left(t_{k-j}\right)$ \\
$\ldots$ & $\ldots$ & $\cdots$ \\
$t_{k}+\Delta L_{i}+\left(\lambda_{i}-1\right) T_{s} \rightarrow t_{k}+L_{i}$ & $t_{k-1} \rightarrow t_{k}$ & $u\left(t_{k-1}\right)$ \\
$t_{k}+L_{i} \rightarrow t$ & $t_{k} \rightarrow t-L_{i}$ & $u\left(t_{k}\right)=u_{k}$ \\
\hline \multicolumn{2}{|c}{ TABLE $\mathrm{I}$}
\end{tabular}

INPUT SEQUENCE DEFINITION.

$$
\begin{aligned}
y_{i}(t) & =y_{p, k} e^{\frac{-\left(t-t_{k}\right)}{\tau_{i}}}+\frac{G_{i}}{\tau_{i}} e^{\frac{-t}{\tau_{i}}} u\left(t_{\left.k-\lambda_{i}-1\right)} \int_{t_{k}}^{t_{k}+\Delta L_{i}} e^{\frac{s}{\tau_{i}}} d s\right. \\
& +\frac{G_{i}}{\tau_{i}} e^{\frac{-t}{\tau_{i}}}\left(\sum_{j=1}^{j=\lambda_{i}} u\left(t_{k-j}\right) \int_{t_{k}+\Delta L_{i}+\left(\lambda_{i}-j\right) T_{s}}^{t_{k}+\Delta L_{i}+\left(\lambda_{i}-j+1\right) T_{s}} e^{\frac{s}{\tau_{i}}} d s\right) \\
& +\frac{G_{i}}{\tau_{i}} e^{\frac{-t}{\tau_{i}}} u\left(t_{k}\right) \int_{t_{k}+L_{i}}^{t} e^{\frac{s}{\tau_{i}}} d s .
\end{aligned}
$$

(20) can be summarized as a linear expression in the optimization argument $u_{k}$ :

$$
\begin{aligned}
& y_{i}(t)=y_{p, k} f_{1 i}\left(\tau_{i}, t_{k}, t\right)+f_{2 i}\left(T_{s}, G_{i}, \tau_{i}, \Delta L_{i}, \lambda_{i}, t_{k}, t, u(\text { past })\right) \\
& +u_{k} f_{3 i}\left(G_{i}, \tau_{i}, L_{i}, t_{k}, t\right),
\end{aligned}
$$

where the $f_{i}$ may be explicitly defined offline and numerically updated online at each time $t_{k}$.

Therefore, replacing first (21) in (9), assuming that the weights $w_{i, k}$ are constant over the prediction horizon, then combining this result with (15) and (16) in (14), the cost function to minimize can be written as:

$J\left(u_{k}\right)=\int_{t_{k}}^{t_{k}+t_{p}}\left(\left(\sum_{i=1}^{N}\left(w_{i, k} y_{i}(t)\right)+e_{k}-y_{k}^{s p}\right)^{2}+w_{u} \Delta u_{k}^{2}\right) d t$

where $t_{p}=\max \left(t_{p i}\right) \forall i$ is each prediction horizon has to be tuned according:

$$
\left\{\begin{aligned}
t_{p i} & =\gamma_{p} * \tau_{i}+L_{i} ; \gamma_{p} \in \mathbb{R}^{+}, \\
\text {e.g.: } \gamma_{p} & =1(63 \% \text { of the dynamics is predicted }) \\
\text { or } \gamma_{p} & =3(95 \% \text { of the dynamics is predicted }) .
\end{aligned}\right.
$$

Based on the step response series (20) of the $N$ linear FOPTD models, the expression (22) is a quadratic one in $u_{k}$ :

$J\left(u_{k}\right)=\beta_{2, k}\left(N, G_{i}, \tau_{i}, L_{i}, t_{p}, w_{u}, w_{i, k}\right) u_{k}^{2}$

$+\beta_{1, k}\left(N, T_{s}, G_{i}, \tau_{i}, L_{i}, t_{p}, \Delta L_{i}, \lambda_{i}, w_{u}, y_{p, k}, y_{k}^{s p}, e_{k}, u(\right.$ past $\left.), w_{i, k}\right) u_{k}$ $+\beta_{0, k}\left(N, T_{s}, G_{i}, \tau_{i}, L_{i}, t_{p}, \Delta L_{i}, \lambda_{i}, w_{u}, y_{p, k}, y_{k}^{s p}, e_{k}, u(\right.$ past $\left.), w_{i, k}\right)$

The $\beta_{i, k}($.$) can be easily explicitly defined offline (see the$ appendix) and are updated at each time $t_{k}$.

Since $J$ is convex in $u_{k}$ (due to the fact that $\beta_{2, k}>0$, by definition), let us then define

$$
u_{k}^{\min }\left(N, T_{s}, G_{i}, \tau_{i}, L_{i}, t_{p}, \Delta L_{i}, \lambda_{i}, w_{u}, y_{p, k}, y_{k}^{s p}, e_{k}, u(\text { past }), w_{i, k}\right)
$$

the solution of the minimization of (24) obtained with the first order optimality at each $t_{k}$ :

$$
\frac{\partial J}{\partial u_{k}}=0 \quad \text { at } u_{k}=u_{k}^{\min }
$$

The calculation of $u_{k}^{\min }$ is then straightforward:

$$
u_{k}^{\min }=\frac{-\beta_{1, k}}{2 \beta_{2, k}}
$$

which leads to the explicit formulation of the solution $u_{k}^{\star}$ of the constrained optimization problem (14):

$$
\left\{\begin{array}{l}
\text { if } u_{\text {inf }} \leq u_{k}^{\text {min }} \leq u_{\text {sup }}: u_{k}^{\star}=u_{k}^{\text {min }} \\
\text { if } u_{k}^{\text {min }} \leq u_{\text {inf }}: u_{k}^{\star}=u_{\text {inf }} \\
\text { if } u_{\text {sup }} \leq u_{k}^{\text {min }}: u_{k}^{\star}=u_{\text {sup }} .
\end{array}\right.
$$

Using (28), the solution is very fast to compute (no online optimization task, no online dynamic model resolution).

\section{F. Simulation results}

The MMPC strategy is then implemented into Simulink and coupled to a detailed experimentally validated model [6]. The engine operation covers a heat flow rate entering into 


\begin{tabular}{lrl}
\hline$K_{i}$ & - & 380 \\
\hline$\delta$ & - & 0.001 \\
\hline$\tau_{\text {filt }}$ & $s$ & 5.33 \\
\hline$\gamma_{p}$ & - & 5 \\
\hline$T_{s}$ & $m s$ & 20 \\
\hline$w_{u}$ & $K^{2} \cdot \mathrm{kg}^{-2} \cdot \mathrm{s}^{-2}$ & $\min \left(G_{i}\right)$ \\
\hline
\end{tabular}

CONTROLLER TUNING PARAMETERS

the WHRS going from 0 to $140 \mathrm{~kW}$ that is divided into $10 \mathrm{~kW}$ zones, hence leading to 14 different triplets of the FOPTD parameters. As seen in figure 3 for two of them, this model structure is validated by comparing complete simulation results of the nonlinear model $(1-2)$ and each SISO FOPTD model (5) identified around an operating point. On both operating points the agreement is good. Model parameters of the FOPTD models change a lot from one operating point to another as it can be seen on figure 4 , hence underlying the nonlinear aspect of the model.
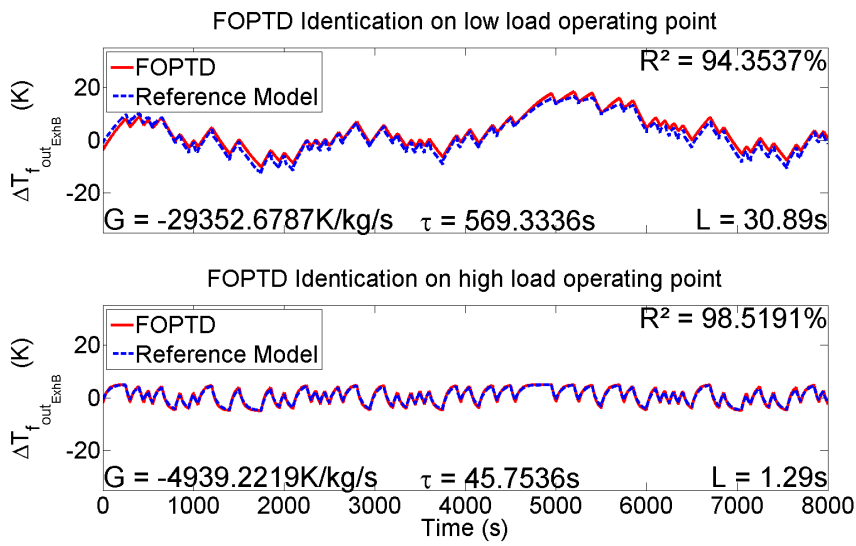

Fig. 3. FOPTD and nonlinear model variations for two operating points.
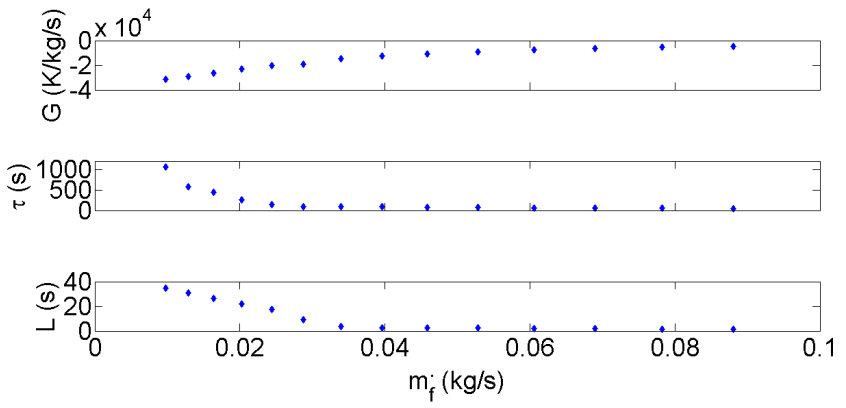

Fig. 4. FOPTD model parameters

The controller is then tested on a validated simulation model [6] and the two weighting schemes are compared. Perfect actuators and sensors are considered and controller tuning parameters are set to obtain the best performance. All values of the convergence matrix $K$ are set to the same value. Table II shows the controller tuning parameters used $\left(i \in\left[\begin{array}{ll}1 & N\end{array}\right]\right)$. The set point is changed during the simulation time to assess the performance as well for load changes as set point variations. Despite the large inputs disturbances (figure 5) of the representative long haul truck driving cycle used, the tracking performance (shown in figure 6) of the
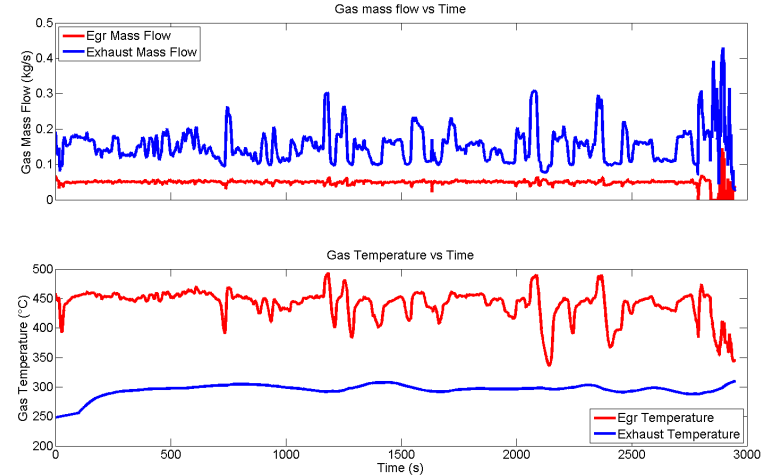

Fig. 5. Input disturbances

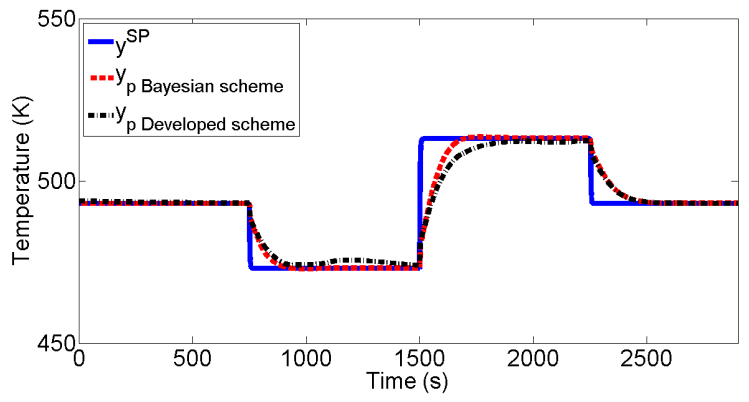

Fig. 6. Tracking performance

controller for the two implemented weighting schemes is good. The Bayesian scheme gives better performance as well in terms of accuracy as in response time. The main reason is the presence of a cut off probability $(\delta)$ which allows here to converge to a single model faster than with the other approach. The developed weighting scheme uses more blending to compute the global model output. In addition to that, the new developed weighting scheme seems less performant to reach the set point when it moves far from the operating point where the 14 FOPTD models have been identified. For both weighting schemes, the time response is quite long compared to the use of a nonlinear model based controller [10], which requires meanwhile the full perfect knowledge of all nonlinear model parameters and an online nonlinear model inversion (which may be no compatible with our integration constraints).

\section{G. Conclusion}

This paper reports an explicit control law based multi-model predictive controller (MMPC). Based on FOPTD models, it is a fast control algorithm since it does not require any online optimization, nor a dynamic model resolution. A weighting scheme for multi linear models approach is also shown and compared to a classical Bayesian weighting scheme. Simulation results developed for a waste heat recovery Rankine based system used in heavy duty trucks, show similar performance to the Bayesian scheme but contrary to this method, the new proposed approach requires less effort in terms of tuning. Currently experimentally validated, the new 
proposed weighting scheme outperforms the Bayesian for a low number of linear models in the data bank. However, for both cases shown here, the time response is longer than with a nonlinear model based controller structure and needs to be improved. In the meantime, the explicit controller presented here is a very fast algorithm and does not require to solve online a dynamic model that might be too time consuming, contrarily to the nonlinear model based approach. The presented controller can be derived either from an available detailed model (1-2), or directly from experiments (without detailed model development). Also, a known set point trajectory into the prediction horizon could be beneficial on this aspect but needs to be designed. Indeed, the knowledge of the future operating conditions is here often hard to get and requires complex calculations. At the time of writing, the strategy proposed has been implemented on the experimental setup and first results are convincing.

\section{H. Appendix}

1) $\beta$. expression:

$$
\begin{aligned}
\beta_{1, k}= & \sum_{i=1}^{N}\left[\beta_{1, k}^{\prime}+\beta_{1, k}^{\prime \prime}+\beta_{1, k}^{\prime \prime \prime}+\beta_{1, k}^{\prime \prime \prime \prime}\right]-2 w_{u} u_{k-1} t_{p}, \\
\beta_{1, k}^{\prime}= & -G_{i} \tau_{i} w_{i, k}{ }^{2} y_{p, k}\left(e^{\frac{L_{i}}{\tau_{i}}}+2 e^{-\frac{t_{p}}{\tau_{i}}}-e^{\frac{L_{i}-2 t_{p}}{\tau_{i}}}-2\right) \\
\beta_{1, k}^{\prime \prime}= & -G_{i}{ }^{2} \tau_{i} u\left(t_{k-\lambda_{i}-1}\right) w_{i, k} e^{-\frac{2 t_{p}}{\tau_{i}}}\left(e^{\frac{\Delta L_{i}}{\tau_{i}}}-1\right) \ldots \\
& \ldots\left(e^{\frac{t_{p}}{\tau_{i}}}-1\right)\left(e^{\frac{L_{i}}{\tau_{i}}}-2 e^{\frac{t_{p}}{\tau_{i}}}+e^{\frac{L_{i}+t_{p}}{\tau_{i}}}\right) \\
\beta_{1, k}^{\prime \prime \prime}= & \sum_{i=1} G_{i}{ }^{2} \tau_{i} u\left(t_{k-j}\right) w_{i, k}{ }^{2} e^{\frac{\Delta L_{i}-t_{p}-j T_{s}+\lambda_{i} T_{s}}{\tau_{i}}} \ldots \\
& \ldots\left(e^{\frac{T_{s}}{\tau_{i}}}-1\right)\left(e^{\frac{L_{i}-t_{p}}{\tau_{i}}}-2\right) \ldots \\
& \ldots-G_{i}{ }^{2} \tau_{i} u\left(t_{k-j}\right) w_{i, k}{ }^{2} e^{\frac{\Delta L_{i}-j T_{s}+\lambda_{i} T_{s}}{\tau_{i}}} \ldots\left(e^{\frac{L_{i}}{\tau_{i}}}-2\right)\left(e^{\frac{T_{s}}{\tau_{i}}}-1\right) \\
\beta_{1, k}^{\prime \prime \prime \prime}= & 2 G_{i} w_{i, k}\left(e_{k}-y s p\right)\left(t_{p}+\tau_{i} e^{\frac{L_{i}-t_{p}}{\tau_{i}}}-\tau_{i} e^{\frac{L_{i}}{\tau_{i}}}\right) \\
\beta_{2, k}= & \frac{G_{i}{ }^{2} w_{i, k}{ }^{2}\left(2 t_{p}+4 \tau_{i} e^{\frac{L_{i}-t_{p}}{\tau_{i}}}-\tau_{i} e^{\frac{2 L_{i}-2 t_{p}}{\tau_{i}}}-4 \tau_{i} e^{\frac{L_{i}}{\tau_{i}}}+\tau_{i} e^{\frac{2 L_{i}}{\tau_{i}}}\right.}{2} \\
+ & w_{u} t_{p}
\end{aligned}
$$

\section{REFERENCES}

[1] C. Sprouse III and C. Depcik, "Review of organic rankine cycles for internal combustion engine exhaust waste heat recovery," Applied Thermal Engineering, vol. 51, no. 12, pp. 711 - 722, 2013.

[2] S. Oomori, H. Ogino, "Waste heat recovery of passenger car using a combination of rankine bottoming cycle and evaporative cooling system," in SAE Technical Paper, no. 930880, SAE International, 03 1993.

[3] A. Boretti, "Recovery of exhaust and coolant heat with r245fa organic rankine cycles in a hybrid passenger car with a naturally aspirated gasoline engine," Applied Thermal Engineering, vol. 36, no. 0, pp. 73 - 77, 2012.

[4] J. Peralez, P. Tona, O. Lepreux, A. Sciarretta, L. Voise, P. Dufour, and M. Nadri, "Improving the control performance of an organic rankine cycle system for waste heat recovery from a heavy-duty diesel engine using a model-based approach," in 52nd Annual IEEE Conference on Decision and Control (CDC), pp. 6830-6836, Dec 2013.

[5] J. Zhang, S. Gao, Y. Chen, and G. Hou, "Multivariable robust control for organic rankine cycle based waste heat recovery systems," in Industrial Electronics and Applications (ICIEA), 2013 8th IEEE Conference on, pp. 85-89, June 2013.

[6] V. Grelet, V. Lemort, T. Reiche, P. Dufour, and M. Nadri, "Waste heat recovery rankine cycle based system modeling for heavy duty trucks fuel saving assessment," in 3rd International Seminar on ORC Power Systems (ORC15), 2015 (Accepted), October 2015.

[7] A. Banerjee, Y. Arkun, B. Ogunnaike, and R. Pearson, "Estimation of nonlinear systems using linear multiple models," AIChE Journal, vol. 43, no. 5, pp. 1204-1226, 1997.

[8] B. Aufderheide and B. Bequette, "Extension of dynamic matrix control to multiple models," Computers and Chemical Engineering, vol. 27, no. 89, pp. 1079 - 1096, 2003.

[9] R. Rao, B. Aufderheide, and B. Bequette, "Multiple model predictive control of hemodynamic variables: an experimental study," in Proceedings of the 1999 American Control Conference (ACC), 1999., vol. 2, pp. 1253-1257 vol.2, Jun 1999.

[10] V. Grelet, P. Dufour, M. Nadri, V. Lemort, and T. Reiche, "Model based control for waste heat recovery rankine cycle system in heavy duty trucks," in 3rd International Seminar on ORC Power Systems (ORC15), 2015 (Accepted), October 2015.

\section{NOMENCLATURE}

\section{Greek letters}

$\alpha \quad$ Heat transfer coefficient $\left(W / m^{2} / K\right)$

$\beta \quad$ Polynomial weight (uSI)

$\delta \quad$ Cut off probability (-)

$\gamma \quad$ Prediction tuning parameter (-)

$\rho \quad$ Density $\left(\mathrm{kg} / \mathrm{m}^{3}\right)$

$\tau \quad$ Time constant $(s)$

\section{Latin letters}

$t_{p}$

$T$

A

e

G

h

$J$

K

$L$

N
Mass flow $(\mathrm{kg} / \mathrm{s})$

Prediction time $(s)$

Sampling time $(s)$

Area $\left(m^{2}\right)$

Modeling error (-)

\begin{tabular}{|c|c|}
\hline$n_{d}$ & $\begin{array}{l}\text { Discretization number } \\
(-)\end{array}$ \\
\hline$P$ & Pressure $(\mathrm{Pa})$ \\
\hline$p$ & $\begin{array}{c}\text { Bayesian recursive } \\
\text { scheme probability }(-)\end{array}$ \\
\hline$T$ & Temperature $(K)$ \\
\hline$t$ & Time $(s)$ \\
\hline$u$ & Input $(-)$ \\
\hline V & Volume $\left(\mathrm{m}^{3}\right)$ \\
\hline$w$ & Weight $(-)$ \\
\hline$X$ & $\begin{array}{l}\text { Developed scheme raw } \\
\text { weight }(-)\end{array}$ \\
\hline$x$ & State $(x)$ \\
\hline$y$ & Output $(-)$ \\
\hline \multicolumn{2}{|c|}{ Sub and superscripts } \\
\hline$a m b$ & Ambient \\
\hline ext & External wall \\
\hline$f$ & Working fluid \\
\hline filt & Filter \\
\hline$g$ & Gas \\
\hline int & Internal wall \\
\hline$k$ & Current \\
\hline$p$ & Process \\
\hline$s p$ & Set point \\
\hline$w$ & Heat exchanger wall \\
\hline
\end{tabular}

FOPTD gain $(K / \mathrm{kg} / \mathrm{s})$

Enthalpy $(J / k g)$

Cost function (-)

Bayesian recursive scheme convergence matrix (-)

FOPTD lag $(s)$

Number of local models $(-)$ 